\title{
Analysing the Cultural Impact of a System
}

\author{
R. STAMPER
}

Ronald Stamper is with The London School of Economics, Houghton Street, London WC2A 2 AE, UK.

An earlier version of this paper was prepared for the Conference Information a Basic Resource for Development, in Frcjus, France, 1983, organized by Data for Development

'SPICER, E.H. (Ed.) (1952). Human problems in technological change. New York: Russell Sage Foundation.

${ }^{2}$ I use the term 'methodology' under protest bowing only to customary usage. It would be better, as in the philosophy of science, to speak of "methods" when referring to specific ways of approaching and solving problems, and to reserve 'methodology' for the comparative and critical study of methods in general; otherwise this vital field of study is nameless.

\begin{abstract}
This paper introduces Evaluation Framing, a method of analysing the impact of an innovation such as a new product, a change to an organization, or more specifically, a new information system. It is based on a taxonomy of cultural patterns of behaviour. The object is to be able to perceive the primary. secondary and more tenuous impacts of an innovation upon these patterns of behaviour in order to detect how the people involved will be affected and how they will feel about the intended change. Their evaluations can, in this manner, be placed in a framework against which design proposals can be assessed.
\end{abstract}

All too often information systems fail. Many fail because the people who are expected to use them, quietly ignore or circumvent the computer-based operations. Sometimes opposition is more vocal but more often the system fails to live up to its promise for reasons that may be difficult to pinpoint except as 'user dissatisfaction'.

When introducing a computer into any organization it is necessary to recognize the effects it is likely to have upon people and the demands it will make upon the organization. These problems are common to all technological innovations in all kinds of societies.

Attempts to introduce new technologies have often failed in a developing economy because no one asked in time what impact the changes would have on the culture of the community. When the agent of change comes from an alien culture (or subculture), there is greater difficulty in asking the right questions but a greater need to do so.' Therefore we need to find systematic ways of formulating appropriate questions, especially when innovations involving high technology take place in the developing world.

In this paper I present a methodology which for some years I have taught to my students who come from both the advanced and the dèveloping economies. The ideas come from anthropology, and I originally formulated them when face-to-face with some difficult information systems problems in a developing country. Subsequently, the ideas proved to be just as valuable when faced with predicting the impacts of technology in a Western environment. In both contexts, the method enables the innovator to explore the likely social consequences of his work with greater accuracy and sensitivity than merely by guesswork and 'feel'.

\section{The MEASUR methodology}

A whole battery of techniques has been under development in a long established research project which aims to produce the next generation of information requirements analysis tools. The resulting methodology ${ }^{2}$ is called 'MEASUR' because it consists of Methods for Eliciting, 
Analysing, Specifying User Requirements, which were developed in the LEGOL/NORMA Project. ${ }^{3}$

The other techniques, in addition to Evaluation Framing, deal with matching the system design effort to the social and economic infrastructure, matching the communications subsystem to the informal exchange of information, and matching the control subsystem to the prevailing ethical practices. Another technique deals with the semantic analysis of the domain of discourse so that the language in the computer system matches the normal language of the users instead of being a stilted formal language invented by the programmers and analysts.

The methodology is supported by a formalism (LEGOL/NORMA) in which the organizational system can be specified. This allows a computer, interpreting the specification, to simulate or prototype the logically implied data-processing functions. In many cases, the system generated in this fashion will support the application. Where it proves not to be efficient enough, the LEGOL/NORMA specification can be used to define the tasks to be performed by a system designed to achieve the required throughput, in the assurance that all the essential business functions will have been defined and tested. The MEASUR techniques, including LEGOL/NORMA constitute a representation of a new generation of methodology for systems analysis and design. Its development, along with software support tools, is underway in a project in which business and government are invited to participate.

The project is based theoretically upon the study of organizations as systems in which signs are used to get work done. The computer and other technological means of storing, transmitting and processing signs are treated as secondary to the primary business tasks to be performed. In the analytical methods and in the specification of the business information system there need be no reference to specific patterns of message flow or data-processing functions. These are treated as logical consequences of the business requirements, modified in their implementation to take account of the characteristics of the available technology. Data flow diagrams, function specification diagrams, data structure diagrams and so on, all of which direct attention to the manipulation of data, have no place in MEASUR until the business problem has been specified. Only at that point need one introduce considerations of data-processing technique. The methods in MEASUR

${ }^{3}$ This project began with funding from IBM, continued with support from two UK Research Councils (SERC and ESRC) and, later, from the Computer Board. It is now receiving support from the Digital Equipment Corporation.

${ }^{4}$ Semiology or semiotics, the theory of signs, has an honorable history dating from the Stoic philosophers of ancient Greece. Its importance is being rediscovered after flourishing in the Middle Ages on account of the advent of information technology. Fortunately we have the insights of the logician Charles Sanders Peirce, who flourished at the turn of the century, and Charles Morris, who published his major works on semiotics and behaviour in the 1930 s and 1940s. Before long semiotics will take its place as the science underpinning the information-based economy of the future, just as physics has underpinned the older economy of physical production that we are leaving. that are used at the outset, such as Evaluation Framing, are intended to bring the analyst close to the business problems that are rooted in the informal human system. Other methods gradually disentangle the problem and introduce formality where it is necessary for specifying a technical system. The result is more likely to be a system (not inevitably a computer system) that fits naturally into its human setting.

\section{The system as a message}

Everything (and I do mean everything) can be seen from two points of view: as a physical thing and as a sign or message. As objects or actions, things have physical properties. As signs, they have semiological ${ }^{4}$ or informational properties. Objects have weight, length, energy, and so on, whilst actions have duration, momentum and so on. Signs (information, in other words) have properties such as meaning, validity, relevance, information content and other quite different properties from those studied by physicists and engineers. This paper explains how 
this simple observation leads to a systematic method of assessing artifacts (including information systems) and activities (including systems innovations) as messages.

Despite the dawning of post-industrial society and the growing dominance of information as the primary resource of the advanced economies, we still fail routinely to analyse problems from the information-centred point of view. The anthropologist, Mary Douglas, drives this point home in her book, The world of goods, ${ }^{5}$ where she argues that goods should not only be seen as objects which satisfy individual, and largely physical needs, but also as messages. To see why, observe that, as individuals, we consume very little in isolation but normally invite others to share or at least notice our acts of consumption. Food we usually take in the company of others and we commonly buy each other drinks, whilst houses, furniture, clothes and cars eloquently mark our status. The scrutiny of our use of other goods reveals that none of them have only physical or biological properties; they all function as signs and, in subtle ways, communicate messages about the relationship of their users to their community. Indeed the world of goods can be seen as a language for 'marking' social relationships. Mary Douglas's argument focuses on. consumer goods, but it could extend to other artifacts that interest us, in particular to the technology transferred to the developing economies.

Information systems are no exception. They transmit messages not only within themselves but also of themselves; thus, they function as messages from their creators to those who use them. The physical view of an information system makes us focus on the volumes of data, speeds of transmission, reliability of components, and adaptability of subsystems. On those issues alone we tend to expend all our design efforts. We neglect the information system as a message in its own right.

We can start by observing that every message conveys some of the knowledge and intentions of a sender to a receiver, so an information system, as a message, will convey the views and intentions of the innovator (usually the management or an administration) to the clients, customers, employees and any other users. Often enough those on the receiving end do not like the message they receive. They may be free, as customers to reject it, or as a captive clientele or as employees, they may have little alternative but to receive it and comply with it superficially, whilst neutralizing as much of it as possible. ${ }^{6}$ Managements would be less likcly to makc these mistakes if they saw their systems as messages, as explained below.

SDOUGLAS, M. AND ISHERWOOD, B. (1980) The world of goods. London: Pengiun. 'Op. cil., Ref. 1. bJORN ANDERSON, N., IIEDBERG, B., MERCER, D., MUMFORD, F. AND SOLE, A. (1979). The impact of systems changes in organisations. Alphen aan den Rijn: Sijhoff and Noordhoff.

\section{Culture and evaluation}

No artifact has value in its own right but it has value conferred upon it by another system. In a business, there is usually a formal system for assigning values, one created and run by the accountants. This will reflect and amplify the value judgements of the owners and managers. The judgements of the suppliers and customers will be expressed through the market, those of the ordinary labour force through the industrial relations systems, whilst the general public influences innovations through the broader political system. Basic to all these is the general cultural system in which all these interested parties are involved.

When looking at how an innovation will be judged one needs to take account of all the different judges, all those who are, or feel themselves 
affected. The weights you give to the values they express will depend upon their relative power and influence. Often the people most directly involved are the ordinary labour force who may have little direct power or make explicit response to the new system. However, their failure to understand, adapt, and restructure their behaviour to accommodate the new system often results in a failed innovation. This adverse cultural response may not be deliberate but it can be overwhelming; it flows from the informal, all-pervasive cultural systems against which all artifacts are measured and evaluated.

The culture is the basic evaluative system. Its judgements are decisive but seldom fully articulated. Management needs an analytical technique to make the likely cultural responses evident. The trade unions or other representatives of the labour force need such a technique to help the people they represent to articulate their demands when faced with technical innovations that will radically alter their clients' working lives. Consumer organizations can help to make the market more effective as an evaluative information system by looking analytically at the cultural dimensions of the products and services they test. Marketing departments of companies might consider adding Evaluation Framing to their other methods of analysis. Our products and our innovations speak to a culture and the culture replies, but it speaks in direct response to actual situations and in a silent language. However, we must learn to anticipate all the cultural messages that will be exchanged in advance of the changes we consider making. Using techniques such as the one explained here, we can begin to articulate these messages in ample time to avoid or prepare for adverse reactions.

\section{How culture imparts significance to change}

A culture consists of behaviour patterns that relate to one another in complex ways. To understand these relationships and to cope with the multiplicity of patterns of behaviour, we require a taxonomy. Various taxonomies have been produced by different anthropologists, ${ }^{7}$ but here we shall adopt the rather simple framework that E.T. Hall introduces in his book, The silent language, ${ }^{8}$ and show how to adapt it to our special purposes. The result is a method for predicting those cultural consequences of innovations that are likely to cause trouble if not perceived in time.

Hall proposes ten categories under which every aspect of a culture can be classified. Table 1 lists them and indicates the kinds of behaviour falling under each heading by supplying a few examples in the context of a familiar organizational question: what status does a person have in the hierarchy?

Notice that the organization tells the new manager about his position (an aspect of association) by using aspects of all the other categories of behaviour. There are dozens of cultural signals adding up to a very clear

${ }^{7}$ For example, Malinowski, B. (1960). A scientific theory of culture and other essays. University of North Carolina Press 1944. Galaxy and Oxford UP. I.Fvi-srknuss, (1968). Structural anthropology. London: Penguin. I.EACH, SIR E.R. (1976). Culture and communication. Cambridge UP.

${ }^{*}$ HALL, F.T. (1959). The silent language. New York: Doubleday. and precise 'statement' about his status. The statement need hardly be articulated in words but it is a powerful message, loaded with feeling.

Table 1 omits one important feature: these categories may be grouped in pairs in a manner that emphasizes the biological significance of our cultural framework. The point is important because it helps to account for the strong feelings induced by any tampering with the cultural system. Start with the second pair, subsistence and bisexuality, which go to the roots of our biological nature. Then the third pair, territoriality 
Table 1. Ten cultural means of conveying the same silent message

(The message: a company tells a new middle manager of his status) Behaviour category aspects ('carrier') The signal

0. Interaction

1. Association

2. Subsistence

3. Bisexuality

4. Territoriality

5. Temporality

6. Learning

7. Play

8. Defence

9. Exploitation
Language, vocal inflections means of communication, posture, gesture, etc.

Community, class, caste, roles, organization, teams, hierarchy, etc.

Physical livelihood. eating, excretion and (indirectly) income working for a living

Differentiation of sexes, marriage, family

Division of space, where things go, where to do things, ownership

Division of time, when to do things, sequence duration, cycles

Education, training, rearing, what gets taught or learned

Recreation, fun, games, art, sport, what is amusing Protection against the elements, other groups, disease and the supernatural

Tools, technology, systems, materials and their uses, skills
Reporting structure, circulation lists for documents, gestures and tones of voice conveying superiority/ subordination Membership of various committees, formal place in the hierarchy, whom to obey and whom to direct

Access to management dining room and washing facilities, generally exempt from manual work, receipt of fairly good salary Middle management predominantly male, display of machismo expected Own medium-sized office, desk, filing cabinet, keys to various parts of company territory Fairly flexible hours of work but always 'on call', three weeks of holiday, who waits on whom

Placed on management development schedule, free to attend short conferences and expected to emulate senior management Invited to join golf club, made familiar with 'in' jokes Expected to wear a dark suit, white overall and hard hat, security cleared, given keys and DB password Dictating machine, amplified phone, bleeper, computer terminal (not the latest) 
and temporality, reflect the 'physical' incompatibility of many activities which must have their separate places and times. The next pair, defence and exploitation, have to do with boundaries, exploitation with the extension of a person or group whilst defence serves to keep the individual or group from being taken over by nature, by other groups or by supernatural forces. Then learning and play: these behaviours nurture growth and discovery. Finally, the first pair on Hall's list, interaction and association, embody social structures and their interconnections which provide the setting for all other behaviours. We can use this classification scheme to understand the cultural reverberations which travel through a society on the impact of new technology.

\section{The voice of culture}

We speak by using the sounds we can make to carry the word patterns. Altering the tension in our vocal chords and the volumes of space in the cavities of the mouth, we modulate the sound in pitch and timbre. Similarly, sound signals are used to modulate the electromagnetic signal from a radio transmitter. Indeed, every time we send any kind of signal, we modulate some 'carrier' which has known and predictable characteristics, to impose upon it the novel pattern that is our signal. The voice of culture speaks in a similar way.

Just as learning to speak words is difficult for the deaf, learning to 'speak' in the 'voice' of a given culture can be difficult for the insensitive person. A normal person acquires a cultural language easily, at least on the scale of personal relationships. On an institutional scale it is less simple. If we stay with friends and sit in their grandfather's favourite chair, his demeanour, if not the remarks of our hosts, will inform us of the message we convey to him by invading his space. Feedback at the personal level is swift. However, at the institutional level feedback is slow and allows time for expensive mistakes to be made before we recognize the errors of our ways. Additionally, we find many people in the institutional context treat such matters in the style of a boorish guest who thinks, 'They invited me here, so I have a right to sit where I like'. It is that kind of closed mind that guarantees an inability to learn to speak acceptably in cultural terms, but it does not guarantee silence. The cultural boor cannot avoid announcing and exposing his character to the wider community who are usually unwillingly forced to notice him. At the institutional level we need every technique that will enable us to anticipate the feedback and so accelerate our learning processes.

The carrier for our cultural messages is the system of cultural norms, the pattern of intricately interrelated behaviours which we regard as the familiar background to everything we do. The dependencies among the cultural norms ensure that a change in one quarter will modulate behaviour elsewhere. Then, because our culture springs from deep within our physical and biological nature, the secondary changes tend to evoke strong emotions. To detect the emotions that will reverberate through a community or an organization, first we should examine each affected area of behaviour in turn. Table l illustrates this in terms of a person's status in an organization. The example has been chosen because questions of status often arouse strong feelings and information systems can easily affect status markers. Each category of behaviour can mark a person's status and on joining the organization as, say, a middle manager, as illustrated here, the silent messages will speak loud and 
clear. (Look down column 3 of Table 1.) Status, as a person understands it emotionally, will consist of this whole repertoire of behaviour which differentiates him from others along that dimension. This first examination of the problem will reveal how a variable in one cultural area (here status which belongs to association) may depend upon behaviour in every other cultural domain.

This analysis has exposed some of the indicators of status that will be read as a message by the incoming middle manager. After a while, these indicators will be the familiar background of his position. They will then have become the carrier which an innovation can modulate. Changes may affect a number of these status indicators, resulting in signals that will be felt as a new message about his status.

To perform this second level of analysis, we note any change in the established hehaviour (possibly resulting from some technical or organizational innovation). Quite unintentionally, these changes may then signal a deeper change which a person or community may perceive as a threat or (rarely) the reverse. In the example, status will seem under threat if any of its markers should change. Thus, access to certain documents $(0)$, appointment to another committee (1), a salary increase (2), inclusion in the directors' group at a night club after a company policy conference (3), a new office carpet (4), no longer being kept waiting by a director (5), an invitation to speak at the company policy conference (6), invited to join a bridge circle of more senior managers (7), higher security status (8), new desk-top computer terminal (9), would all tend to signify promotion even though nothing has been said explicitly on the matter. The secondary analysis has revealed a layer of messages encoded by modulating the accepted cultural norms that were treated as messages in the first analysis.

\section{Modulation of the cultural pattern by an innovation}

We can now begin to see how the cultural 'carrier', with its predictable behaviour, can be modulated in order to transmit quite a powerful emotion-laden signal. The common difficulty is that we too often inadvertently 'say' the wrong thing in this silent language.

In Table 1, we have seen how aspects of every category of behaviour can function as cultural markers of a person's status in a hierarchy (just one aspect of association behaviour). When we attempt to predict the impacts of an innovation, such as the introduction of some new technology or a reorganization of a business, we shall have to investigate the impacts upon all the behaviour categories. In particular, we need to look out for the unintentional impacts. The innovation will be interpreted as a message with the meaning suggested by the enforced changes in behaviour. The analysis applies, of course, not only to status-marking behaviour, but to every one of the ten streams into which culture is subdivided in this model.

This method of analysis applies to any innovation which may have significant effects on a community. As an example consider (Table 2) the messages communicated when a government programme brings a hand pump to a village which has a difficult water supply problem, (nearest water $2 \mathrm{~km}$ on the surface or $20 \mathrm{~m}$ below the surface). We may assume that the pump occupies a central site in the village, acquired from some land-owner; inspection and maintenance by the water authority will ensure a continuing interaction between a previously 
Table 2. Examples of the silent messages conveyed by a government programme providing hand pumps for village drinking water supply in India

Change induced directly

Significance in other cultural areas

(0) New routine communications with water authority for maintenance and inspection. Introduction of new work and concepts concerned with the pump, water quality and officialdom

(01) Someone acquires status through the new responsibilities he or she acquires. Reveals influence of central authority (08) Knowledge of sources of danger to health

(09) A way of disseminating methods of working (machines and

bureaucracy!)

(1) All castes have to use the same water source.

Regular contact with inspector

(2) Domestic water more readily available.

Drinking water chlorinated

(3) Water carrying simplified. New tasks associated with the pump and its maintenance (4) Land for hand pump facility owned by village council. Space near facility not for waste water disposal

(5) Domestic water in all seasons. Quicker to reach water source, but longer queueing time

(6) Some people instructed in simple maintenance

(7) Pump affords new games to the children

(8) Defence against drought. Protection afforded against disease. government policy on caste (14) Increased awareness of wide community outside the village (28) Increased well-being and self respect because cleaning and washing easier

(21) Officialdom can intrude even into the cup one drinks from

(31) Status of women can improve (319) Sex roles open to redefinition in terms of new technology

(41) Collective ownership and responsibility more acceptable (48) Importance conferred on the hand pump facility

(52) Increased well-being

(51) New patterns of association may evolve

(66) Practical value of schooling reinforced

(79) Socialization of children to new technology begins

(88) Influence of deities perceived (89) provided they use the system correctly Caste threatened by need to share facility

(9) Pump priming has to be arranged.

New devices (valves) and materials (plastic washers: not leather!) become familiar (81) Old power structures disrupted by technology (91) New skills may depend on new patterns of collaboration (90) Pump adopted as an analogy and seen as a way of solving other problems

distant bureaucracy and the village. The social changes wrought by this simple technology will affect not only its own acceptability but the attitudes of the villagers to subsequent intrusions by central authority. Most people will benefit by the changes but not everyone to the same extent. In the first stage of the analysis we look for the direct impact which the new systems will have in each area of the village's culture whilst in the second stage we look at the consequence of these primary impacts again in each of the ten areas. 
The examples in Table 2 cover a wide range of effects and illustrate how they can be named (the primary impacts have a one-digit code number, and the secondary ones, a two-digit code). If you consider the examples in Table 2 rather uneven in importance then please accept this as the price of trying to illustrate the whole range of ten behaviour categories. You may consider play as unimportant but, as a matter of fact, play certainly did sometimes adversely affect the tube-well programme in an Indian state; once the drill rig departed, the children had irresistible fun dropping stones down the bore-hole, at some cost to the programme! You may care to add your own thoughts about some of the other 75 or so facets of the problem not considered - there is plenty of analysis to be performed in this example.

The choice of the hand-pump as an illustration emphasizes that the method of Evaluation Framing can be applied outside the domain of information systems which is the focus of this journal. Nevertheless, there is a sense in which the method can be regarded as a tool of information management. We tend to overlook the generation of ideas as a key function in any adaptive organization. Creativity suffers in our (UK 1988) cultural terms from being in the category of behaviour number 7 , play. Automatically it is marked down as self-indulgent, unbusinesslike and unprofitable. Art schools and faculties of humanities are being closed down and our best designers move to countries such as Italy where creativity and style are valued. Evaluation Framing acts as a prop to creative thinking. There is nothing automatic about generating the contents of the numbered cells in the matrix of cultural impacts. Faced with any novel situation, they are the products of imagination. A skilful user of the method will not, of course, rely upon his own imagination but will involve the various groups that will be affected by the change, and he will do so by stimulating their imaginative thinking. (Incidentally, all the novel methods in MEASUR act as aids to creative thinking.) Any manager responsible for the information resources of a company should consider carefully his organization's capacity for creative thinking (not a preserve of Research and Development or Advertising departments). Techniques such as this have wide applicability.

The creative aspect of using Evaluation Framing does not have to eliminate order and disciplined thinking. It is clearly possible to apply the method to problem domains that will be encountered repeatedly (for example, the water-supply problem in developing countries). In these circumstances, the framework can be used to organize an accumulating body of experience without preventing further imaginative thinking. Indeed, the refinement of a domain-specific model will draw attention to the finer points that may have been overlooked initially. This illustrates an important feature of good analytical

"I object strongly to the belief, now current in the 'expert' systems community, that knowledge can be expressed in hundreds of PROLOG rules that are opaque to any user, even opaque to their creators after the passage of a little time. If purchasers of so-called 'expert systems' can be led to believe that knowledge can be put into black boxes and sold to them, they are being deceived themselves. Knowledge is a social construct and it dies if not open to and constantly subjected to criticism and testing. techniques. Not only must they lead the analyst to consider important issues and raise them with the people concerned, but they must provide a systematic way of organizing the information gathered in a framework that simplifies analysis and draws out the important conclusions with a minimum of effort. A good representation technique for a large body of information has another major benefit: it opens the 'knowledge-base' to critical scrutiny. ${ }^{9}$ Methods such as Evaluation Framing are simple, easy to remember and appropriate even for the disciplined use of back-of-the envelope problem-solving, as well as for structuring phases of sophisticated decision-conferencing. The aim in the MEASUR methodology 
has been to discover, as in this case, open yet disciplined methods of grasping the substantive business problem.

\section{The matrix of changes}

A change in one aspect of behaviour, as we have seen, has a potential impact on behaviour on every other area of a culture and also upon some other aspects of the same area. The cascade model (Figure 1) invites us to consider $10 \times 10=100$ possible effects after two stages. These in turn can generate possible effects in each area, so the analysis can continue. How far one goes depends upon the problem and the importance of the reactions of people. The analysis should terminate when it can add no more, when it has played out its role of prompter, focusing the analyst's attention on aspects of the problem he might easily overlook. The potential complexity seems overwhelming but it can be controlled by making the analysis specific to a problem domain.

Hall, whose framework we have adapted, provides a 10 by 10 matrix that he calls 'A map of culture'. In the various cells, he enters aspects of behaviour where one category of behaviour interacts with another. For example the economic aspects of learning (cell 62) draw attention to the rewards for teaching and learning. One can prepare specialized maps of this kind that will accelerate the analysis in a problem domain. The map, or Evaluation Framework for information systems, is partly shown in Tables 3a and 3b where you will notice that bisexuality is subsumed, along with race and other classifications of individuals, under taxonomy.

To understand how to perform the analysis, make use of familiar ideas or correlations. If you know a person is tall then you can guess he is relatively heavy and vice versa. If in a given culture, you are, for example, looking at territoriality (4) you might identify a certain space as a potential source of problems. Given a good knowledge of that culture, you will then be able to state what activities are permitted there or not: Can you, in that space

(0) Speak, sing, shout, ... .

(1) Accommodate a family, a person of high status, a committee

(2) Eat, drink, work ... .

(3) Have women there, only men or both . . .

(4) Subdivide the space, reach other space ...

(5) Find different things happening there at different times,

(6) Are certain kinds of learning (technical, formal or prescriptive, informal or imitative) more likely there

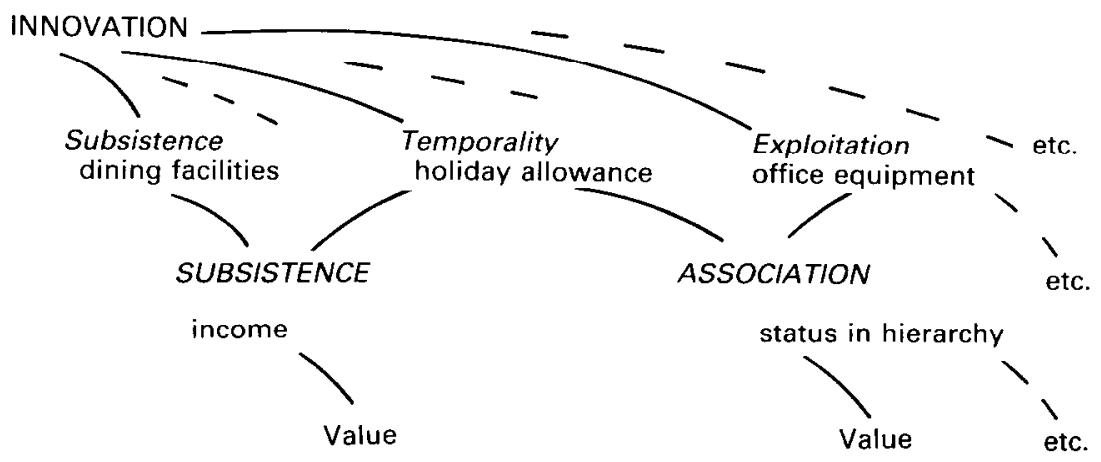

Figure 1. Cascade model of cultural impacts 
(7) Are only certain kinds of play, humour, enjoyment permitted

(8) Are people defended against the elements, against other people, or against spiritual forces

(9) Exploit it in some special way as a kind of tool for growing things, building things, or performing....?

Quite probably an innovation will change the structure at the first level, say by altering the shape of, access to, lighting of.... the space. There will then be knock-on effects at the second level, for example:

(0) It becomes unsuitable for conversation,

(1) It would be appropriate only for a person of low status, . . and so on

through all ten areas of cultural behaviour. Whenever the technology or innovation alters the pattern of correlation between one area of culture and another, then the impact of the change should be estimated at least crudely. The final step is to make some estimate of the likely evaluation of the change that would be ascribed by each of the significant groups or

Table 3a. Headings for the Evaluation Framework

\begin{tabular}{|c|c|c|}
\hline Column A & & Column B \\
\hline 0. Semiological & \multirow{5}{*}{ aspects } & Interaction \\
\hline 1. Organizational & & Association \\
\hline 2. Economic & & Subsistence \\
\hline 3. Taxonomic & & Taxonomy \\
\hline 4. Territorial & & Space \\
\hline 5. Temporal & \multirow[t]{5}{*}{ of } & Time \\
\hline 6. Instructional & & Learning \\
\hline 7. Enjoyment & & Play/creativity \\
\hline 8. Protective & & Defence \\
\hline 9. Exploitational & & Production \\
\hline $\begin{array}{l}\text { Combine an adject } \\
\text { column B }\end{array}$ & with a no & number in \\
\hline
\end{tabular}

Table 3b. Some examples of the cells in the Evaluation Framework for information systems

02: Semiological aspects of Subsistence Currency Information about values

08: Semiological aspects of Defence Indicators of random errors Indicators of deliberate intrusion

13: Organizational aspects of Taxonomy Division of work by sex and race, etc. Provision of facilities for child care

16: Organizational aspects of Learning Training courses Assignment of experienced staff to help others

20: Economic aspects of Interaction Costs of transactions 
Table 3b.

Relevance

Information quantities

29: Economic aspects of Production

Costs of materials and processes

Values of products

32: Taxonomic aspects of Subsistence Influence of sex or race upon income Class-related consumption patterns

37: Taxonomic aspects of Play/Creativity Difference sources of enjoyment for each sex Expectations of creativity for each class

41: Spatial aspects of Association Where different groups meet Spaces reserved for certain officers

45: Spatial aspects of Time

Time zones

Spaces reserved for queues and waiting

59: Temporal aspects of Production When certain jobs can be undertaken Speeds of different tools

53: Temporal aspects of Taxonomy Ages of retirement by sex Staff turnover by class

60: Instructional aspects of Interaction Methods of teaching a programming language Teaching sensitivity to cultural communication

64: Instructional aspects of Space Learning to find one's way about Learning to estimate sizes

78: Enjoyment aspects of Defence Practical jokes 'Hacking' to penetrate other computer systems

71: Enjoyment aspects of Association Friendships formed at work Pleasure found in status

84: Protective aspects of Space Use of partitions, doors etc. Privacy

85: Protective aspects of Time Rest periods Bells, sirens, etc. to partition time

96: Exploitational aspects of Learning Use of teaching machines Teaching skills

97: Exploitational aspects of Play/Creativity Generation of novel products Professional entertainment

persons affected. In this way we can gauge rather better whether a change will be understood and accepted or not.

Once the analysis has penetrated far enough, one can respond by adapting the design or plan to emphasize the desirable features and reduce those which are likely to interfere with established behaviour patterns. This response may be mixed with deliberately disrupting undesirable, established cultural patterns - that might be the aim of some innovations in agriculture or public health for example - but, 
more often than not, the cultural system has to be sustained if the innovation is to be accepted.

\section{Application to information systems}

The same method works if we have to analyse an information system as the cause of the social perturbation. However, certain primary effects will tend to predominate. Interaction must change, of course, and with it association is nearly always affected too. Subsistence and bisexuality behaviours will adjust as the job specifications change. The location of computing terminals and the scheduling of work will impact the areas of territoriality and temporality. Usually learning about such a system requires more formality whilst the formality can take away much of the enjoyment (play) from the established interactions. The new equipment extends the community's exploitation behaviours and it also raises problems of defence. If we treat data stores as a kind of territory. their defence incorporates the ideas of access and privacy in information systems. Table 4, which I shall briefly explain, illustrates the Evaluation Framing applied to an information system.

A brief sketch of the scenario for the example chosen, a system for agricultural loans, is as follows. The setting is the rural area of an East African country. Assume that the traditional local moneylenders play a significant and not always beneficial role in the financing of agriculture. Also assume that a system of farmers' cooperatives exists for the purchase of equipment, seeds and fertilizers and the marketing of produce. In this setting, the government wishes to introduce cheaper loans for farmers with the twin objectives of influencing the quality of agriculture in the country and of shifting financial power from the local moneylenders to a central banking system. The system will operate by making the cooperative responsible for the administration and eventual repayment of the loans, thus giving the local community a stake in the good conduct of each borrower. The cooperative borrows from the central bank and then lends to its members, whilst the government provides guarantecs in exchange for participants accepting the discipline of its system. The cooperative, using a robust microcomputer system, will keep detailed records of each contract and use them to schedule the undertakings entered into by the farmer/borrower; visits to him to instruct, advise and inspect will also take place according to this schedule. This outside interference will justify a very low interest rate designed to buy the cooperation of the agricultural community. Sufficient of the cultural consequences of the system to illustrate the method appear in Table 4 . There are plenty of other points to consider but that task will be left to the reader as an exercise.

\section{A summary and warnings}

By way of summary, here are the key points. First select a simple taxonomy of social behaviour (Hall's tenfold classification, for example). Then for any innovation, classify its main impacts on each of these areas of behaviour. Next, search for secondary effects under each of the headings, taking each main impact as a starting point. Remember that these effects, primary, secondary and even tertiary, can arouse quite strong feelings, and these feelings will determine the acceptability of the 
Table 4. Some possible cultural impacts of an information system to arrange and administer agricultural loans

Main impact Secondary effects

0 : Detailed, strictly formal documentation of the contract required

1: The farming community, through the cooperative, acquires greater collective responsibility, especially for the money borrowed from the central bank

2: Income distribution changes; traditional moneylenders hard hit

\section{3: (No primary effect)}

4: Farms covered by the scheme acquire new significance. Detailed information about them recorded by the system. Loss of privacy
01: The official in the cooperative acquires a powerful role because of the technical competence needed to deal with the new contract document

03: Women qualify as well as men for the official's role

06: The cooperative gives instruction in the system especially in the documentation of contracts

069: Farmers discover the formal contract as an instrument for conducting business

12: Livelihood now dependent upon behaviour of the neighbours to a greater degree

15: Discharge of collective responsibilities depends on a schedule and the meetings and other events become significant items on the calendar

17: The need to know more neighbours rather better encourages wider recreational contact

24: Price of land increases causing disputes over land inheritance

281: Moneylenders (also usually farmers) defend their positions by moving into the bureaucracy of the agricultural loans' systems

40: Increased discussion of the scheme and farms affected

401: The community monitors, collectively and informally, the new agricultural practices introduced via the scheme and learns the most effective ones with further inducement

48: Defence of property rights improved. Privacy lost 
Table 4.

Main impact

5: Detailed schedules for borrowing, repayment, completion of tasks and inspection contained in each contract

6: Primarily the government devised the scheme to induce farmers to learn new methods

7: Possibility of some limited recreational use of the computer

8: System provides, through the government guarantee, some defence against hazards which might cause borrowers to default on a wide scale

9: Contracts and interactions with advisers become tools of practical farming. The computer supports the process
Secondary effects

50: Communications about the contract become prominent in the individual farmer's working cycle

526: Role of planning in good farming practice becomes more widely appreciated

59: Better use made of time for sharing more expensive equipment

61: Organization set up for more adult education in farming and related matters

66: Formal instruction increases

69: Instruction given in the use of new tools, fertilizers and crops

71: Group created for that purpose

80: Some understanding of insurance - reciprocity of duties to obtain protection from a much wider community

81: Role of national government better appreciated

91: Explicit rules understood as a basis for association

92: Information grows in economic importance

94: Ownership becomes a more subtle concept, dependent upon conditions of contracts being met

98: The importance of contracts for defence of one's interests better appreciated

innovation at the social level. Finally, if appropriate to do so, estimate the benefits and costs of the proposed change from the points of view of the relevant interest groups. This simple method of analysis should reveal many potential problems at the design stage.

The method will not guarantee that the systems designer will recognize all the consequences, since how well it works will depend on his insight and sensitivity to the community affected. Moreover, he should not base his conclusions only upon introspection unless he has no access to the actual community. It is always desirable to work with those 
concerned especially if one is not a member of the subculture involved: accountants, doctors, patients and nurses, for example, belong to different subcultures and it is difficult to place oneself in their shoes. Nonetheless, starting the process of design at this point, and dealing with it methodically, will avoid many of the difficulties which needlessly take us by surprise when we introduce new information systems into an organization or, quite generally, any innovation into a community.

Another caveat: this account neglects some important issues you should consider when applying the method. In particular, the interaction between the systems analyst and the affected community requires attention. A participative technique can be used, where the analyst interacts closely in a systematic way with the various ultimate users of the system. Do not neglect the social aspects of the analysis and design process itself.

\section{The future of evaluation framing}

The method has been taught for some years to graduate students of information systems at the London School of Economics. They have been able to grasp the key ideas very quickly and have applied them to an astonishingly wide range of problems varying in scale from the introduction of word processing into an office, to the assessment of the impacts of various government policies such as introducing information technology to a developing country, or closing mental hospitals in favour of community care. The principles of Evaluation Framing are so simple and obviously useful that they spread by word of mouth to graduate students in other departments.

Even at the most superficial level, the understanding of the cultural impacts of technology within this rather simplistic framework (seen through the eyes of an anthropologist) has been found to help the person with a technological background to observe organizational variables more acutely. Perhaps the fact, that Evaluation Framing gives a rather structured, slightly mechanistic introduction to an alien subject that is normally treated discursively accounts for its ready acceptance by people with a computer science background. Of course, they should be encouraged to examine more of the literature and to treat the method critically. The motivation to do this grows as skill in using the methods grows and, with it, a perception of intriguing problems.

Currently, a software support tool for Evaluation Framing is under development. The work forms a part of the LEGOL/NORMA project. The software will form part of a comprehensive and general decisionsupport environment for information requirements analysis incorporating all the MEASUR methodology. Several of these tools, including the one to support Evaluation Framing, are applicable to problems outside the information systems domain. Initially, there will be a special version for the design of information systems but it is hoped to encompass other problem areas, especially the selection of investment projects for developing countries where errors caused by technology failing to fit a culture are common.

Research in this area of methodology will continue and collaboration will always be welcome. 